\title{
On the Compositional Characterization of Complete Fuzzy Pre-orders
}

\author{
Susana Díaz, Bernard De Baets, Susana Montes
}

\begin{abstract}
Complete pre-orders can be characterized in terms of the transitivity of the corresponding strict preference and indifference relations. In this paper, we investigate this characterization in a fuzzy setting. We consider two types of completeness (weak completeness and strong completeness) and decompose a fuzzy pre-order by means of an indifference generator, in particular a Frank t-norm. In the weakly complete case, we identify the strongest type of transitivity of the indifference and strict preference relations in function of the generator used for constructing them. In the strongly complete case, we lay bare a stronger type of transitivity of the strict preference relation. We conclude the paper with a rather negative result: there is no hope to obtain a compositional characterization of weakly complete fuzzy pre-orders, and hence also not of fuzzy pre-orders in general.
\end{abstract}

keyword Frank t-norm, fuzzy pre-order, indifference, strict preference, transitivity

\section{Introduction}

In many applications of relational calculus, transitivity is a desirable, even essential property of the relations involved. A trivial, yet useful observation is the fact that the transitivity of a complete relation is equivalent to the transitivity of its symmetrical and asymmetrical parts. Or, using the language of preference modelling, "the transitivity of a complete large preference relation is characterized by the transitivity of the corresponding indifference and strict preference relations".

This simple observation poses quite a challenging problem when trying to express it in fuzzy relational calculus. There the matter is far more complicated, mainly because of the multiple degrees of freedom involved. First, there is no unique notion of transitivity for fuzzy relations. Second, there is no unique notion of completeness. Third, there is no unique way to decompose a large preference relation. The design choices made in this paper are the following:

(i) We restrict the problem to min-transitive large preference relations, as they have received ample attention before [14]. Moreover, min-transitivity is an essential property in fuzzy relational calculus $[8,12]$. 
(ii) We focus our attention on two popular types of completeness, known as strong and weak completeness, as they are the ones considered before in this problem setting $[4,10]$.

(iii) For the decomposition, we call upon the successful framework of indifference generators developed by De Baets and Fodor [6]. The transitivity of the resulting components will be expressed as precisely as possible by means of appropriate conjunctors.

In the strongly complete case, the choice of the indifference generator is immaterial. In that case, a characterization of min-transitive large preference relations in terms of the corresponding indifference and strict preference relations has been obtained in [10]. This characterization requires not only the transitivity of the corresponding indifference and strict preference relations, but also additional compositional conditions involving both of these components. As far as we know, no other characterizations have been reported on. The mathematical preliminaries and the results mentioned are described in detail in Sections $2-4$.

The transitivity of the indifference and strict preference components of a fuzzy pre-order, i.e. a min-transitive large preference relation, have been presented in [14]. We strengthen these results in the presence of weak completeness: for the indifference relation and a general continuous indifference generator in Section 4; for the strict preference relation and a Frank t-norm as generator in Section 5. We revisit the strongly complete case in Section 6 and conclude in Section 7 with a remarkable impossibility result: no characterization of the type anticipated exists for weakly complete fuzzy pre-orders, i.e. min-transitive weakly complete large preference relation.

\section{Preference structures}

\subsection{Crisp preference structures}

Suppose that a decision maker wants to judge a set of alternatives $A$. Given two alternatives, she can act in one of the following three ways: (i) she clearly prefers one to the other; (ii) the two alternatives are indifferent to her; (iii) she is unable to compare the two alternatives. Accordingly, three (binary) relations on $A$ can be defined: the strict preference relation $P$, the indifference relation $I$ and the incomparability relation $J$. Recall that for a relation $R$ on $A$, its converse is defined as $R^{t}=\{(b, a) \mid(a, b) \in R\}$, its complement as $R^{c}=\{(a, b) \mid(a, b) \notin R\}$ and its dual as $R^{d}=\left(R^{t}\right)^{c}$. One easily verifies that the quadruplet $\left(P, P^{t}, I, J\right)$ establishes a particular partition of $A^{2}$.

Definition 2.1 [25] A preference structure on $A$ is a triplet $(P, I, J)$ of relations on $A$ that satisfy:

(i) $P$ is irreflexive, $I$ is reflexive and $J$ is irreflexive; 
(ii) $P$ is asymmetrical, $I$ and $J$ are symmetrical;

(iii) $P \cap I=\emptyset, P \cap J=\emptyset$ and $I \cap J=\emptyset$;

(iv) $P \cup P^{t} \cup I \cup J=A^{2}$.

Every preference structure can be identified with a unique reflexive relation called large preference relation $R=P \cup I$. This relation leads back to the preference structure in the following way:

$$
(P, I, J)=\left(R \cap R^{d}, R \cap R^{t}, R^{c} \cap R^{d}\right) .
$$

Recall that a relation $Q$ on $A$ is transitive if $((a, b) \in Q \wedge(b, c) \in Q) \Rightarrow$ $(a, c) \in Q$, for any $(a, b, c) \in A^{3}$. Transitivity of $Q$ can also be expressed as $Q \circ Q \subseteq Q$ (with $\circ$ the usual composition of relations). The transitivity of the large preference relation $R$ can be characterized in terms of relational compositions [1].

Theorem 2.1 For any reflexive relation $R$ with corresponding preference structure $(P, I, J)$ it holds that

$$
R \circ R \subseteq R \quad \Leftrightarrow \quad(P \circ P \subseteq P \wedge I \circ I \subseteq I \wedge P \circ I \subseteq P \wedge I \circ P \subseteq P) .
$$

Recall that a reflexive and transitive relation is called a pre-order. The foregoing theorem can therefore be seen as a characterization of a pre-order in terms of the associated indifference and strict preference relations. A complete pre-order $R$ (i.e. $R \cup R^{t}=A^{2}$ ) is called a weak order. For a weak order, this characterization can be simplified as follows. Note that in this case $J=\emptyset$.

Theorem 2.2 For any complete (reflexive) relation $R$ with corresponding preference structure $(P, I, \emptyset)$ it holds that

$$
R \circ R \subseteq R \quad \Leftrightarrow \quad(P \circ P \subseteq P \wedge I \circ I \subseteq I) .
$$

Definition 2.1 can be written in the following minimal way, by identifying a relation with its characteristic mapping [7]: $I$ is reflexive and symmetrical, and for any $(a, b) \in A^{2}$ it holds that $P(a, b)+P^{t}(a, b)+I(a, b)+J(a, b)=$ 1. Classical preference structures can therefore also be considered as Boolean preference structures, employing 1 and 0 for describing presence or absence of strict preference, indifference and incomparability.

\subsection{Additive fuzzy preference structures}

In fuzzy preference modelling, the notions of strict preference, indifference and incomparability are a matter of degree. These degrees can take values between 0 and 1 and fuzzy relations are used for capturing them. The intersection of fuzzy relations is usually defined pointwisely based on some $t$-norm, i.e. an increasing, commutative and associative binary operation on $[0,1]$ with neutral element 1. The three most important t-norms are the minimum operator $T_{\mathbf{M}}(x, y)=$ 
$\min (x, y)$, the algebraic product $T_{\mathbf{P}}(x, y)=x y$ and the Eukasiewicz t-norm $T_{\mathbf{L}}(x, y)=\max (x+y-1,0)$. The minimum operator is the greatest t-norm; the smallest t-norm is the drastic product $T_{\mathbf{D}}$ defined by

$$
T_{\mathbf{D}}(x, y)= \begin{cases}0 & , \text { if } \max (x, y)<1 \\ \min (x, y) & , \text { otherwise }\end{cases}
$$

Similarly, the union of fuzzy relations is based on a t-conorm, i.e. an increasing, commutative and associative binary operation on $[0,1]$ with neutral element 0. T-norms and t-conorms come in dual pairs: to any t-norm $T$ there corresponds a t-conorm $S$ through the relationship $S(x, y)=1-T(1-x, 1-y)$. For the above three t-norms this yields the maximum operator $S_{\mathbf{M}}(x, y)=$ $\max (x, y)$, the probabilistic sum $S_{\mathbf{P}}(x, y)=x+y-x y$ and the Eukasiewicz t-conorm (bounded sum) $S_{\mathbf{L}}(x, y)=\min (x+y, 1)$. For more information on t-norms and t-conorms, we refer to [22].

The definition of a fuzzy preference structure has been discussed for many years $[11,18,26,27]$. The assignment principle, expressing that for any pair of alternatives $(a, b)$ the decision maker is allowed to assign at least one of the degrees $P(a, b), P(b, a), I(a, b)$ and $J(a, b)$ freely in the unit interval, leads to a fuzzification of Definition 2.1 with intersection based on the Eukasiewicz tnorm and union based on the Łukasiewicz t-conorm. This definition admits the same short formulation as the classical one: a triplet $(P, I, J)$ of fuzzy relations on $A$ is a fuzzy preference structure on $A$ if and only if $I$ is reflexive $(I(a, a)=1$ for any $a \in A)$ and symmetrical, and for any $(a, b) \in A^{2}$ it holds that $P(a, b)+P^{t}(a, b)+I(a, b)+J(a, b)=1$. This expression justifies the adjective additive. Note that $P$ is irreflexive, and that $J$ is irreflexive and symmetrical.

Another difficult point has been how to construct such a structure from a reflexive fuzzy relation. The most recent and most successful approach is that of De Baets and Fodor based on (indifference) generators [6].

Definition 2.2 A generator $i$ is a commutative $[0,1]^{2} \rightarrow[0,1]$ mapping that satisfies $T_{\mathbf{L}} \leq i \leq T_{\mathbf{M}}$.

Note that a generator always has neutral element 1 . With a given generator $i$, we associate the $[0,1]^{2} \rightarrow[0,1]$ mappings $p(x, y)=x-i(x, y)$ and $j(x, y)=$ $i(x, y)-(x+y-1)$. The triplet $(p, i, j)$ is called a generator triplet. For any reflexive fuzzy relation $R$ on $A$ it holds that the triplet $(P, I, J)$ of fuzzy relations on $A$ defined by:

$$
\begin{aligned}
& P(a, b)=p(R(a, b), R(b, a))=R(a, b)-i(R(a, b), R(b, a)), \\
& I(a, b)=i(R(a, b), R(b, a)), \\
& J(a, b)=j(R(a, b), R(b, a))=i(R(a, b), R(b, a))-(R(a, b)+R(b, a)-1),
\end{aligned}
$$

is an additive fuzzy preference structure on $A$ such that $R(a, b)=P(a, b)+$ $I(a, b)$. The fuzzy relation $R$ is again called the large preference relation.

A generator triplet $(p, i, j)$ is called monotone if: (i) $p$ is increasing in the first and decreasing in the second argument; (ii) $i$ is increasing in both arguments; 
(iii) $j$ is decreasing in both arguments. The following theorem emphasizes the importance of quasi-copulas [6]. Quasi-copulas are witnessing increasing popularity in fuzzy logic (see e.g. $[5,21]$ ): a binary operation $Q:[0,1]^{2} \rightarrow[0,1]$ is called a quasi-copula if it has neutral element 1 , is increasing and fulfills the 1-Lipschitz property [19]:

$$
\left|Q\left(x_{1}, y_{1}\right)-Q\left(x_{2}, y_{2}\right)\right| \leq\left|x_{1}-x_{2}\right|+\left|y_{1}-y_{2}\right|
$$

for any $\left(x_{1}, x_{2}\right),\left(y_{1}, y_{2}\right) \in[0,1]^{2}$. For any quasi-copula $Q$ it holds that $T_{\mathbf{L}} \leq$ $Q \leq T_{\mathrm{M}}$

Theorem 2.3 A generator triplet $(p, i, j)$ is monotone if and only if $i$ is a commutative quasi-copula.

Imposing additional restrictions leads to particular generator triplets. For instance, the only generator triplets $(p, i, j)$ for which it holds that the mappings $p(x, 1-y), i(x, y)$ and $j(1-x, 1-y)$ are all t-norms, are determined by a Frank t-norm, i.e. $i=T_{\lambda}^{\mathbf{F}}$ for some $\lambda \in[0, \infty][6]$. In the latter case it holds that $p(x, y)=T_{1 / \lambda}^{\mathbf{F}}(x, 1-y)$ and $j(x, y)=T_{\lambda}^{\mathbf{F}}(1-x, 1-y)$. In this paper, general results will be complemented by results that are specific for these popular generators.

For the sake of completeness, we recall that the Frank t-norms are given by

$$
T_{\lambda}^{\mathbf{F}}(x, y)= \begin{cases}T_{\mathbf{M}}(x, y) & , \text { if } \lambda=0 \\ T_{\mathbf{P}}(x, y) & , \text { if } \lambda=1 \\ T_{\mathbf{L}}(x, y) & , \text { if } \lambda=\infty \\ \log _{\lambda}\left(1+\frac{\left(\lambda^{x}-1\right)\left(\lambda^{y}-1\right)}{\lambda-1}\right) & , \text { otherwise }\end{cases}
$$

Recall that given an automorphism $\phi$ and a t-norm $T$, the $\phi$-transform $T^{\phi}$ of $T$ is the t-norm defined by $T^{\phi}(x, y)=\phi^{-1}(T(\phi(x), \phi(y))$. For a strict Frank t-norm $T_{\lambda}^{\mathbf{F}}$, i.e. $\left.\lambda \in\right] 0, \infty\left[\right.$, there exists an automorphism $\phi_{\lambda}$ (also called multiplicative generator) such that $T_{\lambda}^{\mathbf{F}}(x, y)=\phi_{\lambda}^{-1}\left(\phi_{\lambda}(x) \cdot \phi_{\lambda}(y)\right)=\left(T_{\mathbf{P}}\right)^{\phi_{\lambda}}(x, y)$. More explicitly, for any $x \in[0,1]$ it holds that $\phi_{1}(x)=x$ and $\phi_{\lambda}(x)=\left(\lambda^{x}-1\right) /(\lambda-1)$, for any $\lambda \in] 0,1[\cup] 1, \infty[$.

\section{$3 \quad$ Fuzzy weak orders}

\subsection{Transitivity of fuzzy relations}

The transitivity of fuzzy relations is traditionally defined in terms of a t-norm. Here, we work with a very general class of binary operations called conjunctors [13]. This class encompasses the classes of t-norms and quasi-copulas.

Definition 3.1 A conjunctor $f$ is an increasing binary operation on $[0,1]$ that coincides on $\{0,1\}^{2}$ with the Boolean conjunction. 
The smallest conjunctor $c_{\mathbf{S}}$ takes value 1 in $(1,1)$ and value 0 elsewhere; the greatest conjunctor $c_{\mathrm{G}}$ takes value 0 when one of its arguments is 0 , and value 1 elsewhere. Often additional properties will be imposed, such as having neutral element 1 . The smallest conjunctor with neutral element 1 is the t-norm $T_{\mathbf{D}}$, while $T_{\mathbf{M}}$ is the greatest such conjunctor.

Definition 3.2 Consider a conjunctor $f$. A fuzzy relation $Q$ on $A$ is called $f$-transitive if for any $(a, b, c) \in A^{3}$ it holds that $f(Q(a, b), Q(b, c)) \leq Q(a, c)$.

Note that if $f_{1} \leq f_{2}$, then $f_{2}$-transitivity implies $f_{1}$-transitivity. A very popular type of transitivity is $T_{\mathbf{M}}$-transitivity. Defining the composition $Q_{1} \circ_{f} Q_{2}$ of two fuzzy relations $Q_{1}$ and $Q_{2}$ w.r.t. a conjunctor $f$ by

$$
Q_{1} \circ_{f} Q_{2}(a, c)=\sup _{b} f\left(Q_{1}(a, b), Q_{2}(b, c)\right),
$$

still allows us to use the shorthand $Q \circ_{f} Q \subseteq Q$ to denote $f$-transitivity.

A reflexive and $T_{\mathbf{M}}$-transitive fuzzy relation is called a fuzzy pre-order [28].

\subsection{Complete fuzzy pre-orders}

Given a t-conorm $S$, a fuzzy relation $R$ on $A$ is called $S$-complete [18] if it holds that $S(R(a, b), R(b, a))=1$, for any $(a, b) \in A^{2}$. Two particular cases have received ample attention in the literature: $S_{\mathrm{M}}$-complete fuzzy relations are called strongly complete, while $S_{\mathbf{L}}$-complete fuzzy relations are called weakly complete. Note that weak completeness is sometimes called connectedness [4]. Obviously, the greater the t-conorm, the weaker the completeness condition. In particular, strong completeness implies weak completeness. Another way of expressing completeness is through the use of the $T$-linearity condition $[3,20]$, with $T$ a t-norm: a fuzzy relation $R$ on $A$ is called $T$-linear if it holds that $N_{T}(R(a, b)) \leq R(b, a)$, for any $(a, b) \in A^{2}$, with $N_{T}$ the residual negator of $T$ (see [23] for more information on the residual negator). For the relationship of this notion with $S$-completeness, we refer to [3]. Strong completeness is at the core of weak $T$-orders (i.e. strongly complete and $T$-transitive fuzzy relations, with $T$ a t-norm). An overview of various representation theorems for such weak $T$-orders is given in [2]; weak $T_{\mathbf{M}}$-orders on a finite universe are discussed in [9].

In previous work [14], we have fully characterized the transitivity of the indifference and strict preference parts of a fuzzy pre-order, obtained by decomposing it additively by means of a Frank t-norm as generator. We recall some older results related to $S$-complete fuzzy pre-orders:

(i) If a fuzzy pre-order is strongly complete, then De Baets et al. [10] showed that the choice of the generator is irrelevant: the corresponding additive fuzzy preference structure is given by $I(a, b)=\min (R(a, b), R(b, a))$, $P(a, b)=1-R(b, a)$ and $J(a, b)=0$. Moreover, $I$ and $P$ are $T_{\mathbf{M}}$-transitive as well. 
(ii) If the fuzzy pre-order is weakly complete, then Dasgupta and Deb [4] have proven that when using the minimum operator as generator, $I$ and $P$ are $T_{\mathbf{M}}$-transitive as well. They also provide counterexamples showing that when using the Eukasiewicz t-norm as generator, neither the $T_{\mathbf{M}^{-}}$ transitivity of $I$ nor the $T_{\mathbf{M}^{-}}$-transitivity of $P$ holds in general.

In view of its additive formulation, i.e. $R(a, b)+R(b, a) \geq 1$, weak completeness is of particular interest in the context of additive fuzzy preference structures. In order not to confuse between the adjective 'weak' used both for referring to the type of completeness and the type of fuzzy pre-order, we will stick to the term "weakly complete fuzzy pre-order". As the (restrictive) strongly complete case is closed, we focus in this paper on the weakly complete case and try to lay bare the correspondence between the transitivity of the indifference and strict preference parts of a weakly complete fuzzy pre-order and the generator used for constructing them.

\section{Transitivity of the indifference relation}

\subsection{General results}

The following theorem generalizes a result proven in [14] for bisymmetric increasing generators only.

Theorem 4.1 Consider an increasing generator $i$. For any reflexive fuzzy relation $R$ with corresponding indifference relation $I=i\left(R, R^{t}\right)$ it holds that

$$
R \text { is } T_{\mathbf{M}} \text {-transitive } \Rightarrow I \text { is i-transitive. }
$$

Moreover, this is the strongest result possible.

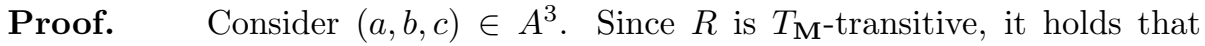
$\min (R(a, b), R(b, c)) \leq R(a, c)$ and $\min (R(c, b), R(b, a)) \leq R(c, a)$. We can distinguish the following four cases: (i) $R(a, c) \geq R(a, b)$ and $R(c, a) \geq R(b, a)$; (ii) $R(a, c) \geq R(b, c)$ and $R(c, a) \geq R(c, b)$; (iii) $R(a, c) \geq R(a, b)$ and $R(c, a) \geq$ $R(c, b)$; (iv) $R(a, c) \geq R(b, c)$ and $R(c, a) \geq R(b, a)$.

In case (i), resp. (ii), it holds that $I(a, c) \geq I(a, b)$, resp. $I(a, c) \geq I(b, c)$. Since $i \leq T_{\mathbf{M}}$, it holds that $I(a, c) \geq \min (I(a, b), I(b, c)) \geq i(I(a, b), I(b, c))$.

In cases (iii) and (iv), it holds that $\min (R(a, c), R(c, a)) \geq \min (I(a, b), I(b, c))$ and $\max (R(a, c), R(c, a)) \geq \max (I(a, b), I(b, c))$. Since $i$ is commutative and increasing, it follows that

$$
\begin{aligned}
I(a, c) & =i(R(a, c), R(c, a)) \\
& =i(\min (R(a, c), R(c, a)), \max (R(a, c), R(c, a))) \\
& \geq i(\min (I(a, b), I(b, c)), \max (I(a, b), I(b, c))) \\
& =i(I(a, b), I(b, c)) .
\end{aligned}
$$


As we have proven in Theorem 6 of [16] that the transitivity of $I$ is bounded by $i$, this is the strongest result possible.

\subsection{The weakly complete case}

In this subsection we start from a weakly complete fuzzy pre-order and identify the transitivity of the corresponding indifference relation. Obviously, this case is intermediate between the general case (no completeness condition) and the strongly complete case. We prove that when imposing weak completeness, a stronger type of transitivity than in Theorem 4.1 can be guaranteed. The characterization of this type of transitivity requires a particular class of binary operations. Let $\Delta=\left\{(x, y) \in[0,1]^{2} \mid x+y>1\right\}$. Given a binary operation $h:[0,1]^{2} \rightarrow[0,1]$, we construct the binary operation $f^{h}:[0,1]^{2} \rightarrow[0,1]$ as follows:

$$
f^{h}(x, y)=\left\{\begin{array}{cl}
h(x, y) & , \text { if }(x, y) \in \Delta, \\
\inf _{z \in[x, 1-y]} h(z, 1-z) & , \text { otherwise } .
\end{array}\right.
$$

In particular, it holds that $f^{h}(x, 1-x)=h(x, 1-x)$. Since for $(x, y) \in \Delta^{c}$ it holds that $(x, y) \leq(z, 1-z)$ for any $z \in[x, 1-y]$, it follows that $f^{h} \geq h$ whenever $h$ is increasing. Note that the t-norms $T_{\mathbf{L}}$ and $T_{\mathbf{M}}$ are not affected by this transformation: if $h \in\left\{T_{\mathbf{L}}, T_{\mathbf{M}}\right\}$, then $f^{h}=h$.

\section{Lemma 4.2}

(i) If $h$ is a conjunctor, then so is $f^{h}$.

(ii) If h has neutral element 1 , then so has $f^{h}$.

(iii) If $h$ is commutative, then so is $f^{h}$.

(iv) If $h$ is a generator, then so is $f^{h}$.

(v) If $h$ is a quasi-copula, then so is $f^{h}$.

\section{Proof.}

(i) By definition $f^{h}=h$ on $\{(1,1),(0,1),(1,0)\}$. In addition, $f^{h}(0,0)=$ $\inf _{z \in[0,1]} h(z, 1-z) \leq h(0,1)=0$. Consider $y_{1} \leq y_{2}$ and $x$ in $[0,1]$. Since $h$ is increasing, it suffices to consider $\left(x, y_{1}\right) \in \Delta^{c}$. If $\left(x, y_{2}\right) \in \Delta^{c}$, then $\left[x, 1-y_{1}\right] \supseteq\left[x, 1-y_{2}\right]$ and

$$
f^{h}\left(x, y_{1}\right)=\inf _{z \in\left[x, 1-y_{1}\right]} h(z, 1-z) \leq \inf _{z \in\left[x, 1-y_{2}\right]} h(z, 1-z)=f^{h}\left(x, y_{2}\right) .
$$

If $\left(x, y_{2}\right) \in \Delta$, then

$$
f^{h}\left(x, y_{1}\right) \leq h(x, 1-x) \leq h\left(x, y_{2}\right)=f^{h}\left(x, y_{2}\right) .
$$

The case $f^{h}\left(y_{1}, x\right) \leq f^{h}\left(y_{2}, x\right)$ is analogous. 
(ii) Immediate.

(iii) Immediate.

(iv) In view of (iii), and the fact that $h$ takes values between $T_{\mathbf{L}}$ and $T_{\mathbf{M}}$, it suffices to show that $f^{h}$ takes values between these two t-norms on $\Delta^{c}$. Let $(x, y) \in \Delta^{c}$. Obviously, $f^{h}(x, y) \geq 0=T_{\mathbf{L}}(x, y)$. On the other hand,

$$
f^{h}(x, y) \leq h(x, 1-x) \leq x \quad \text { and } \quad f^{h}(x, y) \leq h(y, 1-y) \leq y .
$$

Hence, $f^{h}(x, y) \leq \min (x, y)$.

(v) In view of (i)-(ii), it suffices to show that $f^{h}$ fulfills the 1-Lipschitz property. Note that due to continuity (implied by the 1-Lipschitz property), the infimum in (2) is always attained. Since the 1-Lipschitz property of a binary operation is equivalent to the 1-Lipschitz property of its partial mappings, and since $f^{h}$ is increasing, it suffices to show that $f^{h}\left(x, y_{2}\right)-$ $f^{h}\left(x, y_{1}\right) \leq y_{2}-y_{1}$ for any $x \in[0,1]$ and any $y_{1}<y_{2} \in[0,1]$. The other argument can be dealt with in the same way. We distinguish the following cases:

(a) If $x+y_{1}>1$, then the inequality is trivially fulfilled due to the 1-Lipschitz property of $h$.

(b) If $x+y_{1} \leq 1$ and $x+y_{2}>1$, then $f^{h}\left(x, y_{2}\right)=h\left(x, y_{2}\right)$. Since $f^{h}\left(x, y_{1}\right) \geq h\left(x, y_{1}\right)$, it follows that

$$
f^{h}\left(x, y_{2}\right)-f^{h}\left(x, y_{1}\right) \leq h\left(x, y_{2}\right)-h\left(x, y_{1}\right) \leq y_{2}-y_{1} .
$$

(c) Finally, consider the case $x+y_{2} \leq 1$. If $f^{h}\left(x, y_{1}\right)=f^{h}\left(x, y_{2}\right)$, then there is nothing to prove. Else, $f^{h}\left(x, y_{1}\right)=h(z, 1-z)$ for some $\left.z \in] 1-y_{2}, 1-y_{1}\right]$ and consequently $f^{h}\left(x, y_{1}\right)=f^{h}\left(1-y_{2}, y_{1}\right) \geq h(1-$ $\left.y_{2}, y_{1}\right)$. By construction, it also holds that $f^{h}\left(x, y_{2}\right) \leq h\left(1-y_{2}, y_{2}\right)$. Therefore,

$$
f^{h}\left(x, y_{2}\right)-f^{h}\left(x, y_{1}\right) \leq h\left(1-y_{2}, y_{2}\right)-h\left(1-y_{2}, y_{1}\right) \leq y_{2}-y_{1} .
$$

The following theorem largely generalizes the results of Dasgupta and Deb [4] for the generators $i=T_{\mathbf{L}}$ and $i=T_{\mathbf{M}}$.

Theorem 4.3 Consider an increasing generator $i$. For any weakly complete reflexive fuzzy relation $R$ with corresponding indifference relation $I=i\left(R, R^{t}\right)$ it holds that

$$
R \text { is } T_{\mathbf{M}} \text {-transitive } \Rightarrow I \text { is } f^{i} \text {-transitive. }
$$

Moreover, when $i$ is continuous this is the strongest result possible.

Note that this result is indeed stronger than the general result, as it holds that $f^{i} \geq i$. Lemma 4.2 implies when a commutative quasi-copula $i$ is used as 
generator, then also $f^{i}$ is a commutative quasi-copula (in that case, it is also the strongest result possible).

Proof. We distinguish again the four cases considered in the proof of Theorem 4.1. In cases (i) and (ii), it holds that $I(a, c) \geq \min (I(a, b), I(b, c))$. Due to Lemma 4.2, $f^{i}$ is also a generator, and hence $f^{i} \leq T_{\mathbf{M}}$. We then have $I(a, c) \geq f^{i}(I(a, b), I(b, c))$.

In cases (iii) and (iv), it holds that $\min (R(a, c), R(c, a)) \geq \min (I(a, b), I(b, c))$ and $\max (R(a, c), R(c, a)) \geq \max (I(a, b), I(b, c))$. In view of Theorem 4.1, it suffices to prove that $f^{i}(I(a, b), I(b, c)) \leq I(a, c)$ whenever $(I(a, b), I(b, c)) \in \Delta^{c}$. We distinguish two more subcases:

(a) If $\min (R(a, c), R(c, a)) \leq 1-\max (I(a, b), I(b, c))$, then

$$
\min (R(a, c), R(c, a)) \in[\min (I(a, b), I(b, c)), 1-\max (I(a, b), I(b, c))] .
$$

The weak completeness of $R$ implies $\max \left(R, R^{t}\right) \geq 1-\min \left(R, R^{t}\right)$, it then follows that

$$
\begin{aligned}
& i(\min (R(a, c), R(c, a)), \max (R(a, c), R(c, a))) \\
& \geq i(\min (R(a, c), R(c, a)), 1-\min (R(a, c), R(c, a))) \\
& \geq f^{i}(\min (I(a, b), I(b, c)), \max (I(a, b), I(b, c))) .
\end{aligned}
$$

(b) If $\min (R(a, c), R(c, a))>1-\max (I(a, b), I(b, c))$, then

$$
\begin{aligned}
& i(\min (R(a, c), R(c, a)), \max (R(a, c), R(c, a))) \\
& \geq i(1-\max (I(a, b), I(b, c)), \max (I(a, b), I(b, c))) \\
& \geq f^{i}(\min (I(a, b), I(b, c)), \max (I(a, b), I(b, c))) .
\end{aligned}
$$

Since $i$ and $f^{i}$ are commutative, it follows that $I(a, c)=i(R(a, c), R(c, a)) \geq$ $f^{i}(I(a, b), I(b, c))$.

It remains to be shown that no stronger result can be obtained when the generator is continuous. Consider a conjunctor $f$ such that $f(x, y)>f^{i}(x, y)$ for some $(x, y) \in] 0,1]^{2}$. Assume first that $(x, y) \in \Delta$. Consider the reflexive fuzzy relation $R$ on $A=\{a, b, c\}$ given by

\begin{tabular}{c|ccc}
$R$ & $a$ & $b$ & $c$ \\
\hline$a$ & 1 & $x$ & $x$ \\
$b$ & 1 & 1 & 1 \\
$c$ & $y$ & $y$ & 1
\end{tabular}

The fuzzy relation $R$ is weakly complete and $T_{\mathrm{M}}$-transitive, but the corresponding indifference relation generated by means of $i$ is not $f$-transitive. Indeed, it holds that

$$
I(a, c)=i(x, y)=f^{i}(x, y)<f(x, y)=f(I(a, b), I(b, c)) .
$$


Next, consider $(x, y) \in \Delta^{c}$. Assume, without loss of generality, that $x \leq y$ and consider the reflexive fuzzy relation $R$ on $A=\{a, b, c\}$ given by

\begin{tabular}{c|ccc}
$R$ & $a$ & $b$ & $c$ \\
\hline$a$ & 1 & $z_{0}$ & $z_{0}$ \\
$b$ & $\alpha$ & 1 & $\beta$ \\
$c$ & $1-z_{0}$ & $1-z_{0}$ & 1
\end{tabular}

where $z_{0} \in[x, 1-y]$ is such that $i\left(z_{0}, 1-z_{0}\right)=\min \{i(z, 1-z) \mid z \in[x, 1-y]\}$, and $\alpha \geq 1-z_{0}$ and $\beta \geq z_{0}$ are chosen such that $i\left(z_{0}, \alpha\right)=x$ and $i\left(1-z_{0}, \beta\right)=y$. The existence of $z_{0}, \alpha$ and $\beta$ follows from the continuity of $i$ and the fact that, for instance for $\alpha$, it holds $i\left(z_{0}, 1-z_{0}\right) \leq i(x, 1-x) \leq x \leq z_{0}=i\left(z_{0}, 1\right)$.

The fuzzy relation $R$ is weakly complete and $T_{\mathbf{M}}$-transitive, but the corresponding indifference relation generated by means of $i$ is not $f$-transitive. Indeed, it holds that

$$
I(a, c)=i\left(z_{0}, 1-z_{0}\right)=f^{i}(x, y)<f(x, y)=f(I(a, b), I(b, c)) .
$$

When using a Frank t-norm as generator, we can write $f^{i}$ explicitly.

Lemma 4.4 Consider the automorphism $\phi_{\lambda}$ corresponding to the Frank -norm $\left.T_{\lambda}^{\mathbf{F}}, \lambda \in\right] 0, \infty\left[\right.$. The function $h_{\lambda}:[0,1] \rightarrow[0,1]$ defined by

$$
h_{\lambda}(z)=\phi_{\lambda}(z) \phi_{\lambda}(1-z)
$$

is continuous, symmetric w.r.t. 0.5 , strictly increasing on $[0,0.5]$ and strictly decreasing on $[0.5,1]$.

Proof. For $\lambda=1, h_{1}(z)=z(1-z)$ and the proof is trivial. Consider $\lambda \in] 0,1[\cup] 1, \infty[$, then

$$
h_{\lambda}(z)=\frac{\lambda^{z}-1}{\lambda-1} \frac{\lambda^{1-z}-1}{\lambda-1} \quad \text { and } \quad h_{\lambda}^{\prime}(z)=\frac{\ln \lambda}{(\lambda-1)^{2}}\left(\lambda^{1-z}-\lambda^{z}\right) .
$$

(i) If $\lambda<1$, then $\ln \lambda<0$ and the function $\lambda^{t}$ is strictly decreasing for $t \in[0,1]$. Then $h_{\lambda}^{\prime}(z)>0$ for $z<0.5$ and $h_{\lambda}^{\prime}(z)<0$ for $z>0.5$.

(ii) If $\lambda>1$, then $\ln \lambda>0$ and the function $\lambda^{t}$ is strictly increasing for $t \in[0,1]$. Then $h_{\lambda}^{\prime}(z)>0$ for $z<0.5$ and $h_{\lambda}^{\prime}(z)<0$ for $z>0.5$.

Hence, $h_{\lambda}$ is strictly increasing on $[0,0.5$ [ and strictly decreasing on $\left.] 0.5,1\right]$, for any $\lambda \in] 0, \infty\left[\right.$. Since $h_{\lambda}$ is continuous, $h_{\lambda}$ is strictly increasing on $[0,0.5]$ and strictly decreasing on $[0.5,1]$.

In order to simplify the notation, we use the shorthand $f^{\lambda}$ for $f^{T_{\lambda}^{\mathbf{F}}}$.

Corollary 4.5 For any weakly complete reflexive fuzzy relation $R$ with corresponding indifference relation I generated by means of $T_{\lambda}^{\mathbf{F}}, \lambda \in[0, \infty]$, it holds that

$$
R \text { is } T_{\mathbf{M}} \text {-transitive } \Rightarrow I \text { is } f^{\lambda} \text {-transitive, }
$$


with $f^{\lambda}$ defined by

$$
f^{\lambda}(x, y)=T_{\lambda}^{\mathbf{F}}(\min (x, y), \max (1-\min (x, y), \max (x, y))) .
$$

Moreover, these are the strongest results possible.

Proof. Consider $i=T_{\lambda}^{\mathbf{F}}$ with $\lambda \in[0, \infty]$. Assume, without loss of generality, that $x \leq y$, then we need to prove that $f^{\lambda}(x, y)=T_{\lambda}^{\mathbf{F}}(x, \max (1-x, y))$. If $(x, y) \in \Delta$, then $f^{\lambda}(x, y)=T_{\lambda}^{\mathbf{F}}(x, y)=T_{\lambda}^{\mathbf{F}}(x, \max (1-x, y))$. If $(x, y) \in \Delta^{c}$, then we need to prove that $f^{\lambda}(x, y)=T_{\lambda}^{\mathbf{F}}(x, 1-x)$. Since $f^{1}=T_{\mathbf{M}}$ and $f^{\infty}=T_{\mathbf{L}}$, this equality trivially holds for $\lambda \in\{0, \infty\}$. Consider $\lambda \in] 0, \infty[$, then we need to show that

$$
f^{\lambda}(x, y)=\min _{z \in[x, 1-y]} T_{\lambda}^{\mathbf{F}}(z, 1-z)=T_{\lambda}^{\mathbf{F}}(x, 1-x) .
$$

It holds that $T_{\lambda}^{\mathbf{F}}(z, 1-z)=\phi_{\lambda}^{-1}\left(\phi_{\lambda}(z) \phi_{\lambda}(1-z)\right)$. Since $\phi_{\lambda}^{-1}$ is an automorphism, it is strictly increasing and it holds that

$$
\begin{aligned}
\min _{z \in[x, 1-y]} \phi_{\lambda}^{-1}\left(\phi_{\lambda}(z) \phi_{\lambda}(1-z)\right) & =\phi_{\lambda}^{-1}\left(\min _{z \in[x, 1-y]} \phi_{\lambda}(z) \phi_{\lambda}(1-z)\right) \\
& =\phi_{\lambda}^{-1}\left(\min _{z \in[x, 1-y]} h_{\lambda}(z)\right) .
\end{aligned}
$$

It now suffices to show that $\min _{z \in[x, 1-y]} h_{\lambda}(z)=h_{\lambda}(x)$. Since $x \leq y$, it holds that $[x, 1-y] \subseteq[x, 1-x]$ and

$$
h_{\lambda}(x) \geq \min _{z \in[x, 1-y]} h_{\lambda}(z) \geq \min _{z \in[x, 1-x]} h_{\lambda}(z) .
$$

Due to Lemma 4.4, it holds that $\min _{z \in[x, 1-x]} h_{\lambda}(z)=h_{\lambda}(x)$, which concludes the proof.

Since $T_{\lambda}^{\mathbf{F}}$ is continuous for all $\lambda \in[0, \infty]$, Theorem 4.3 guarantees that the result mentioned holds and it is the strongest possible.

In general, it holds that $f^{\lambda} \geq T_{\lambda}^{\mathbf{F}}$, while $f^{0}=T_{\mathbf{M}}$ and $f^{\infty}=T_{\mathbf{L}}$. The inequality is strict for $\lambda \in] 0, \infty\left[\right.$. The operation $f^{\lambda}$ is a commutative generator. Only the associativity property is lacking for turning it into a t-norm.

Proposition 4.6 The operation $f^{\lambda}$ is not a $t$-norm for any $\left.\lambda \in\right] 0, \infty[$.

Proof. Consider the function $H_{\lambda}:[0,1] \rightarrow[0,1]$ defined by

$$
H_{\lambda}(t)=\phi_{\lambda}^{-1}\left(\phi_{\lambda}(t) \phi_{\lambda}(1-t)\right)=\phi_{\lambda}^{-1}\left(h_{\lambda}(t)\right) .
$$

Since $h_{\lambda}$ is strictly increasing on $[0,0.5]$ and strictly decreasing on $[0.5,1]$, and $\phi_{\lambda}^{-1}$ is strictly increasing on $[0,1], H_{\lambda}$ is also strictly increasing on $[0,0.5]$ and strictly decreasing on $[0.5,1]$. Moreover, for any $t \in] 0,1[$ it holds that

$$
H_{\lambda}(t)=\phi_{\lambda}^{-1}\left(\phi_{\lambda}(t) \phi_{\lambda}(1-t)\right)<\phi_{\lambda}^{-1}\left(\phi_{\lambda}(t)\right)=t .
$$


Now consider $x, y$ and $z$ such that $z=0.5>y>x>H_{\lambda}(y)$. Since for any $a<b \leq 0.5$ it holds that $f^{\lambda}(a, b)=T_{\lambda}^{\mathbf{F}}(a, 1-a)=H_{\lambda}(a)$, we find

$$
\begin{aligned}
& f^{\lambda}\left(f^{\lambda}(x, y), z\right)=f^{\lambda}\left(H_{\lambda}(x), z\right)=H_{\lambda}\left(H_{\lambda}(x)\right) \\
& f^{\lambda}\left(x, f^{\lambda}(y, z)\right)=f^{\lambda}\left(x, H_{\lambda}(y)\right)=H_{\lambda}\left(H_{\lambda}(y)\right) .
\end{aligned}
$$

Since $H_{\lambda}$ is strictly increasing on $[0,0.5]$ and $x<y$, it follows that

$$
H_{\lambda}\left(H_{\lambda}(x)\right)<H_{\lambda}\left(H_{\lambda}(y)\right),
$$

which proves that $f^{\lambda}$ is not associative.

This result emphasizes the importance of working with conjunctors: the class of t-norms is not sufficiently rich to describe the transitivity of indifference relations.

We conclude this section with a remarkable result. The Frank family is not only a parametric family of t-norms, it is also a parametric family of copulas (although then usually a different parametrization is used) [24]. It is therefore worthwhile to investigate whether the operations $f^{\lambda}$ are also copulas. In fact, we will show a more general result: for any copula $C$, the operation $f^{C}$ is also a copula. To that end, we recall some necessary notions. A binary operation $C:[0,1]^{2} \rightarrow[0,1]$ is called copula if it has absorbing element 0 , neutral element 1 and is 2-increasing [24]. The latter means that for any rectangle $\left[x, x^{\prime}\right] \times\left[y, y^{\prime}\right] \subseteq$ $[0,1]^{2}$, its $C$-volume is positive, i.e.

$$
V_{C}\left(\left[x, x^{\prime}\right] \times\left[y, y^{\prime}\right]\right)=C(x, y)+C\left(x^{\prime}, y^{\prime}\right)-C\left(x, y^{\prime}\right)-C\left(x^{\prime}, y\right) \geq 0 .
$$

Any copula is a quasi-copula and for any copula it holds that $T_{\mathbf{L}} \leq C \leq T_{\mathbf{M}}$. A copula can also be characterized as a 2-increasing conjunctor with neutral element 1 . Volumes of rectangles are additive, in the sense that for all $0 \leq x_{1} \leq$ $x_{2} \leq x_{3} \leq 1$ and all $\left[y, y^{\prime}\right] \subseteq[0,1]$ it holds that

$$
V_{C}\left(\left[x_{1}, x_{2}\right] \times\left[y, y^{\prime}\right]\right)+V_{C}\left(\left[x_{2}, x_{3}\right] \times\left[y, y^{\prime}\right]\right)=V_{C}\left(\left[x_{1}, x_{3}\right] \times\left[y, y^{\prime}\right]\right) .
$$

Proposition 4.7 If $h$ is a copula, then also the operation $f^{h}$ is a copula.

Proof. In view of Lemma 4.2, it suffices to show that $f^{h}$ is 2-increasing. Consider a rectangle $B$ in $[0,1]^{2}$. Three cases need to be distinguished: $B$ is located in $\Delta$, in $\Delta^{c}$ or covers part of $\Delta$ and $\Delta^{c}$. In the latter case, however, $B$ can always be decomposed into two rectangles located in $\Delta$ or $\Delta^{c}$, and a square of the type $\left[x, x^{\prime}\right] \times\left[1-x^{\prime}, 1-x\right]$. Due to the additivity of volumes, it is therefore sufficient to consider the following three cases:

(i) For a rectangle $B=\left[x, x^{\prime}\right] \times\left[y, y^{\prime}\right]$ with $(x, y) \in \Delta$, the volume $V_{f^{h}}(B)$ is trivially positive, as $f^{h}=h$ on $\Delta$ and $h$ is 2-increasing.

(ii) For a square $B=\left[x, x^{\prime}\right] \times\left[1-x^{\prime}, 1-x\right]$, it holds that

$$
V_{f^{h}}(B)=f^{h}\left(x, 1-x^{\prime}\right)+h\left(x^{\prime}, 1-x\right)-h(x, 1-x)-h\left(x^{\prime}, 1-x^{\prime}\right) .
$$

Since $f^{h} \geq h$, it follows that $V_{f^{h}}(B) \geq V_{h}(B) \geq 0$. 
(iii) Finally, we consider a rectangle $B=\left[x, x^{\prime}\right] \times\left[y, y^{\prime}\right]$ with $\left(x^{\prime}, y^{\prime}\right) \in \Delta^{c}$. We denote by $z_{1}, z_{2}, z_{3}$ and $z_{4}$ the smallest values (due to continuity, the infimum is always attained) such that $f^{h}\left(x^{\prime}, y^{\prime}\right)=h\left(z_{1}, 1-z_{1}\right), f^{h}\left(x^{\prime}, y\right)=$ $h\left(z_{2}, 1-z_{2}\right), f^{h}\left(x, y^{\prime}\right)=h\left(z_{3}, 1-z_{3}\right)$ and $f^{h}(x, y)=h\left(z_{4}, 1-z_{4}\right)$. Since $[x, 1-y]=\left[x, 1-y^{\prime}\right] \cup\left[x^{\prime}, 1-y\right]$, it either holds that $z_{4}=z_{3}$ and $f^{h}(x, y)=$ $f^{h}\left(x, y^{\prime}\right)$ or $z_{4}=z_{2}$ and $f^{h}(x, y)=f^{h}\left(x^{\prime}, y\right)$. In the first case, the volume of $B$ is given by $V_{f^{h}}(B)=f^{h}\left(x^{\prime}, y^{\prime}\right)-f^{h}\left(x^{\prime}, y\right)$, while in the second case $V_{f^{h}}(B)=f^{h}\left(x^{\prime}, y^{\prime}\right)-f^{h}\left(x, y^{\prime}\right)$. Since $h$ is increasing, $f^{h}$ is also increasing, and hence $V_{f^{h}}(B) \geq 0$.

\section{Transitivity of the strict preference relation}

\subsection{Known results}

The transitivity of a strict preference relation is in general bounded by the transitivity of the reflexive fuzzy relation from which it is generated [16]. When applying a Frank t-norm to a fuzzy pre-order, we have been able to identify the strongest type of transitivity that can be guaranteed for the corresponding strict preference relation [14]. In that study, an important role was attributed to the nilpotent minimum [17]. This left-continuous t-norm, usually denoted as $T_{\mathbf{n M}}$, is defined by

$$
T_{\mathbf{n M}}(x, y)= \begin{cases}\min (x, y) & , \text { if }(x, y) \in \Delta \\ 0 & , \text { otherwise }\end{cases}
$$

To any automorphism $\left.\phi_{\lambda}, \lambda \in\right] 0, \infty[$, we have associated the automorphism $\varphi_{\lambda}=\phi_{\lambda}^{-1}\left(\sqrt{\phi_{\lambda}}\right)$ [15]. Completing this family with $\varphi_{0}=\lim _{\lambda \rightarrow 0} \varphi_{\lambda}$ and $\varphi_{\infty}=$ $\lim _{\lambda \rightarrow \infty} \varphi_{\lambda}$ leads to the family $\left(\varphi_{\lambda}\right)_{\lambda \in[0, \infty]}$ of $[0,1] \rightarrow[0,1]$ mappings given by

$$
\varphi_{\lambda}(x)=\left\{\begin{array}{cl}
x & , \text { if } \lambda=0, \\
\log _{\lambda}\left(\sqrt{\left.\frac{\lambda^{x}-1}{\lambda-1}(\lambda-1)+1\right)}\right. & , \text { if } \lambda \in] 0,1[\cup] 1, \infty[, \\
\sqrt{x} & , \text { if } \lambda=1, \\
\frac{x+1}{2} \chi_{] 0,1]}(x) & , \text { if } \lambda=\infty .
\end{array}\right.
$$

Note that we use the notation $\chi_{B}$ for the indicator function of a set $B$.

Similarly, we can consider the family of inverse automorphisms $\varphi_{\lambda}^{-1}, \lambda \in$ ] $0, \infty\left[\right.$, and complete with $\varphi_{0}^{-1}=\lim _{\lambda \rightarrow 0} \varphi_{\lambda}^{-1}$ and $\varphi_{\infty}^{-1}=\lim _{\lambda \rightarrow \infty} \varphi_{\lambda}^{-1}$. This yields the 
family $\left(\varphi_{\lambda}^{-1}\right)_{\lambda \in[0, \infty]}$ of $[0,1] \rightarrow[0,1]$ mappings given by

$$
\varphi_{\lambda}^{-1}(x)= \begin{cases}\log _{\lambda}\left(1+\frac{\left(\lambda^{x}-1\right)^{2}}{\lambda-1}\right) & , \text { if } \lambda \in] 0,1[\cup] 1, \infty[, \\ x & , \text { if } \lambda=0, \\ x^{2} & , \text { if } \lambda=1, \\ (2 x-1) \chi_{[0.5,1]}(x) & , \text { if } \lambda=\infty .\end{cases}
$$

Theorem 5.1 [14] For any reflexive fuzzy relation $R$ with corresponding strict preference relation $P=R-T_{\lambda}^{\mathbf{F}}\left(R, R^{t}\right), \lambda \in[0, \infty]$, it holds that

$$
R \text { is } T_{\mathbf{M}} \text {-transitive } \quad \Rightarrow \quad P \text { is } T_{\mathbf{n M}}^{\varphi_{1 / \lambda}} \text {-transitive, }
$$

where

$$
T_{\mathbf{n M}}^{\varphi_{1 / \lambda}}(x, y)= \begin{cases}\min (x, y) & , \text { if } \varphi_{1 / \lambda}(x)+\varphi_{1 / \lambda}(y)>1 \\ 0 & , \text { otherwise }\end{cases}
$$

Moreover, these are the strongest results possible.

For $\lambda \in\left[0, \infty\left[\right.\right.$ it holds that $T_{\mathbf{n M}}^{\varphi_{\lambda}}$ is the $\varphi_{\lambda}$-transform of the nilpotent minimum $T_{\mathbf{n M}}$, in particular $T_{\mathbf{n M}}^{\varphi_{0}}=T_{\mathbf{n M}}$. Although $\varphi_{\infty}$ is not an automorphism, it does hold that $T_{\mathbf{n M}}^{\varphi_{\infty}}$ is a t-norm, namely $T_{\mathbf{M}}$ itself. Hence, the family $\left(T_{\mathbf{n M}}^{\varphi_{\lambda}}\right)_{\lambda \in[0, \infty]}$ is a t-norm family as well, ranging from the nilpotent minimum operator to the minimum operator. Note that if $\lambda_{1} \leq \lambda_{2}$, then $\varphi_{\lambda_{1}} \leq \varphi_{\lambda_{2}}$, hence this family gradually increases between $T_{\mathrm{nM}}$ and $T_{\mathbf{M}}$.

\subsection{The weakly complete case}

The above theorem does not exclude that for strict preference relations generated from a specific class of reflexive fuzzy relations a stronger type of transitivity can be obtained. We will explore this possibility for the class of weakly complete reflexive fuzzy relations and a Frank t-norm as generator. We consider three cases: the case $i=T_{\mathbf{L}}$ in Theorem 5.2, the case $\left.i=T_{\lambda}^{\mathbf{F}}, \lambda \in\right] 0, \infty[$ in Theorem 5.3, and the case $i=T_{\mathbf{M}}$ in Theorem 5.4.

Theorem 5.2 For any weakly complete reflexive fuzzy relation $R$ with corresponding strict preference relation $P$ generated by means of $T_{\mathbf{L}}$ it holds that

$$
R \text { is } T_{\mathbf{M}} \text {-transitive } \Rightarrow P \text { is } g^{\infty} \text {-transitive, }
$$

where

$$
g^{\infty}(x, y)= \begin{cases}\max (x, y) & , \text { if }(x, y) \in \Delta, \\ 0 & , \text { otherwise. }\end{cases}
$$

Moreover, this is the strongest result possible. 
Proof. By definition it holds that $P(a, b)=\min (R(a, b), 1-R(b, a))$. The weak completeness of $R$ then implies that $P(a, b)=1-R(b, a)$. In view of the definition of $g^{\infty}$, it suffices to consider those triplets $(a, b, c)$ that satisfy $P(a, b)+$ $P(b, c)>1$ and prove that $P(a, c) \geq \max (P(a, b), P(b, c))$. It then follows that $R(c, b)=1-P(b, c)<P(a, b)$ and $R(b, a)=1-P(a, b)<P(b, c)$ and hence $R(a, b) \geq P(a, b)>R(c, b)$ and $R(b, c) \geq P(b, c)>R(b, a)$. The $T_{\mathbf{M}^{-} \text {-transitivity }}$ of $R$ then implies that $R(c, b) \geq R(c, a)$ and $R(b, a) \geq R(c, a)$. Combining the foregoing, we obtain $R(c, a) \leq \min (R(c, b), R(b, a))$. Due to the $T_{\mathrm{M}}$-transitivity, this inequality turns into the equality $R(c, a)=\min (R(c, b), R(b, a))$. Consequently, $1-R(c, a)=1-\min (R(c, b), R(b, a))=\max (1-R(c, b), 1-R(b, a))$, or equivalently, $P(a, c)=\max (P(a, b), P(b, c))$.

It remains to be shown that no stronger result can be obtained. Consider a conjunctor $g$ such that $g(x, y)>g^{\infty}(x, y)$ for some $\left.\left.(x, y) \in\right] 0,1\right]^{2}$. Assume, without loss of generality, that $x \leq y$ and consider the following reflexive fuzzy relation $R$ on $A=\{a, b, c\}$ given by

\begin{tabular}{c|ccc}
$R$ & $a$ & $b$ & $c$ \\
\hline$a$ & 1 & $x$ & $y$ \\
$b$ & $1-x$ & 1 & $(1-x) \chi_{\Delta^{c}}(x, y)+y \chi_{\Delta}(x, y)$ \\
$c$ & $1-y \chi_{\Delta}(x, y)$ & $x \chi_{\Delta^{c}}(x, y)+(1-y) \chi_{\Delta}(x, y)$ & 1
\end{tabular}

The fuzzy relation $R$ is weakly complete and $T_{\mathbf{M}}$-transitive, but the corresponding strict preference relation $P$ generated by means of $T_{\mathbf{L}}$ is not $g$-transitive. Indeed, it holds that

$$
P(a, c)=g^{\infty}(x, y)<g(x, y) \leq g(P(a, b), P(b, c)) .
$$

This completes the proof.

Note that $g^{\infty}>T_{\mathrm{M}}$ in some points and is therefore not a t-norm.

Theorem 5.3 For any weakly complete reflexive fuzzy relation $R$ with corresponding strict preference relation $P$ generated by means of $\left.T_{\lambda}^{\mathbf{F}}, \lambda \in\right] 0, \infty[$, it holds that

$$
R \text { is } T_{\mathbf{M}} \text {-transitive } \Rightarrow P \text { is } g^{\lambda} \text {-transitive, }
$$

where

$$
g^{\lambda}(x, y)= \begin{cases}0 & \text {,if } \varphi_{1 / \lambda}(x)+\varphi_{1 / \lambda}(y) \leq 1 \\ \min (x, y) & , \text { if } \varphi_{1 / \lambda}(x)+\varphi_{1 / \lambda}(y)>1 \\ \text { and } \max (x, y) \leq \max \left(\varphi_{1 / \lambda}(\min (x, y)), 1-\varphi_{1 / \lambda}(\min (x, y))\right) & \text {, otherwise. }\end{cases}
$$

Moreover, these are the strongest results possible.

Proof. Let us verify that $g^{\lambda}$ is a conjunctor. Indeed, it holds that $g^{\lambda}(0,0)=$ $g^{\lambda}(0,1)=g^{\lambda}(1,0)=0$ and $g^{\lambda}(1,1)=\min (1,1)=1$. Next, we show that $g^{\lambda}$ is increasing. Since $g^{\lambda}$ is commutative, it suffices to consider $y_{1} \leq y_{2}$ and $x$ such that $\varphi_{1 / \lambda}(x)+\varphi_{1 / \lambda}\left(y_{1}\right)>1$, and hence also $\varphi_{1 / \lambda}(x)+\varphi_{1 / \lambda}\left(y_{2}\right)>1$. 
(i) If $\max \left(x, y_{2}\right)>\max \left(\varphi_{1 / \lambda}\left(\min \left(x, y_{2}\right)\right), 1-\varphi_{1 / \lambda}\left(\min \left(x, y_{2}\right)\right)\right)$, then

$$
g^{\lambda}\left(x, y_{2}\right)=\varphi_{1 / \lambda}^{-1}\left(\max \left(x, y_{2}\right)\right)>\min \left(x, y_{2}\right) \geq \min \left(x, y_{1}\right)
$$

and

$$
g^{\lambda}\left(x, y_{2}\right)=\varphi_{1 / \lambda}^{-1}\left(\max \left(x, y_{2}\right)\right) \geq \varphi_{1 / \lambda}^{-1}\left(\max \left(x, y_{1}\right)\right),
$$

which implies that $g^{\lambda}\left(x, y_{2}\right) \geq g^{\lambda}\left(x, y_{1}\right)$.

(ii) If $\max \left(x, y_{2}\right) \leq \max \left(\varphi_{1 / \lambda}\left(\min \left(x, y_{2}\right)\right), 1-\varphi_{1 / \lambda}(\min (x, y))\right)$, then it holds that $g^{\lambda}\left(x, y_{2}\right)=\min \left(x, y_{2}\right)$. If also $g^{\lambda}\left(x, y_{1}\right)=\min \left(x, y_{1}\right)$, the proof is trivial. Assume therefore that $\max \left(x, y_{1}\right)>\max \left(\varphi_{1 / \lambda}\left(\min \left(x, y_{1}\right)\right), 1-\right.$ $\left.\varphi_{1 / \lambda}\left(\min \left(x, y_{1}\right)\right)\right)$, whence $g^{\lambda}\left(x, y_{1}\right)=\varphi_{1 / \lambda}^{-1}\left(\max \left(x, y_{1}\right)\right)$. Suppose that $x<y_{1} \leq y_{2}$, then $y_{1}>\max \left(\varphi_{1 / \lambda}(x), 1-\varphi_{1 / \lambda}(x)\right) \geq y_{2}$, a contradition. Hence, $x \geq y_{1}$ and $x>\max \left(\varphi_{1 / \lambda}\left(y_{1}\right), 1-\varphi_{1 / \lambda}\left(y_{1}\right)\right)$. We now only need to show that $\min \left(x, y_{2}\right) \geq \varphi_{1 / \lambda}^{-1}(x)$. If $x \leq y_{2}$, the inequality clearly holds since $\phi_{1 / \lambda}(x) \geq \phi_{1 / \lambda}^{2}(x)$. If $y_{2} \leq x$, then $x \leq \max \left(\varphi_{1 / \lambda}\left(y_{2}\right), 1-\varphi_{1 / \lambda}\left(y_{2}\right)\right)$. Since $x>1-\varphi_{1 / \lambda}\left(y_{1}\right) \geq 1-\varphi_{1 / \lambda}\left(y_{2}\right)$, it then follows that $x \leq \varphi_{1 / \lambda}\left(y_{2}\right)$.

Next we prove the implication. Consider $(a, b, c) \in A^{3}$. Assume, without loss of generality, that $P(a, b) \leq P(b, c)$. Since the $T_{\mathrm{M}}$-transitivity of $R$ implies the $T_{\mathbf{n M}}^{\varphi_{1 / \lambda}}$-transitivity of $P$ (Theorem 5.1), it suffices to consider the case

$$
\begin{gathered}
\varphi_{1 / \lambda}(P(a, b))+\varphi_{1 / \lambda}(P(b, c))>1, \\
P(b, c)>\max \left(\varphi_{1 / \lambda}(P(a, b)), 1-\varphi_{1 / \lambda}(P(a, b))\right)
\end{gathered}
$$

and prove that $\varphi_{1 / \lambda}^{-1}(P(b, c)) \leq P(a, c)$.

Since $R$ is weakly complete, it holds that

$$
\begin{aligned}
P(a, b) & =\phi_{1 / \lambda}^{-1}\left(\phi_{1 / \lambda}(R(a, b)) \cdot \phi_{1 / \lambda}(1-R(b, a))\right) \\
& \geq \phi_{1 / \lambda}^{-1}\left(\phi_{1 / \lambda}(1-R(b, a)) \cdot \phi_{1 / \lambda}(1-R(b, a))\right), \\
P(a, b) & =\phi_{1 / \lambda}^{-1}\left(\phi_{1 / \lambda}(R(a, b)) \cdot \phi_{1 / \lambda}(1-R(b, a))\right) \\
& \leq \phi_{1 / \lambda}^{-1}\left(\phi_{1 / \lambda}(R(a, b)) \cdot \phi_{1 / \lambda}(R(a, b))\right) .
\end{aligned}
$$

This implies that $R(b, a) \geq 1-\varphi_{1 / \lambda}(P(a, b))$ and $R(a, b) \geq \varphi_{1 / \lambda}(P(a, b))$.

By definition it holds that $P(b, c) \leq 1-R(c, b)$.

(i) If $R(c, b)<R(c, a)$, then the $T_{\mathbf{M}}$-transitivity of $R$ implies that $R(c, b) \geq$ $R(a, b) \geq \varphi_{1 / \lambda}(P(a, b))$ and

$$
P(b, c) \leq 1-R(c, b) \leq 1-\varphi_{1 / \lambda}(P(a, b)),
$$

a contradiction.

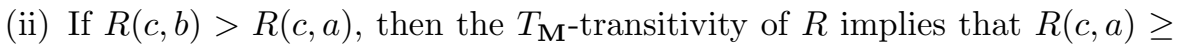
$R(b, a) \geq 1-\varphi_{1 / \lambda}(P(a, b))$ and

$$
P(b, c) \leq 1-R(c, b)<1-R(c, a) \leq \varphi_{1 / \lambda}(P(a, b)),
$$

again a contradiction. 
(iii) If $R(c, b)=R(c, a)$, then

$$
P(b, c) \leq 1-R(c, b)=1-R(c, a) \leq R(a, c) .
$$

This implies that

$$
P(a, c)=T_{1 / \lambda}^{\mathbf{F}}(R(a, c), 1-R(c, a)) \geq T_{1 / \lambda}^{\mathbf{F}}(P(b, c), P(b, c))=\varphi_{1 / \lambda}^{-1}(P(b, c)) .
$$

It remains to be shown that no stronger result can be obtained. Consider a conjunctor $g$ such that $g(x, y)>g^{\lambda}(x, y)$ for some $\left.\left.(x, y) \in\right] 0,1\right]^{2}$. Assume, without loss of generality, that $x \leq y$.

(i) If $\left(\varphi_{1 / \lambda}(x), \varphi_{1 / \lambda}(y)\right) \in \Delta^{c}$, consider the reflexive fuzzy relation $R$ on $A=$ $\{a, b, c\}$ given by

\begin{tabular}{c|ccc}
$R$ & $a$ & $b$ & $c$ \\
\hline$a$ & 1 & $\varphi_{1 / \lambda}(x)$ & $\min \left(\varphi_{1 / \lambda}(x), \varphi_{1 / \lambda}(y)\right)$ \\
$b$ & $1-\varphi_{1 / \lambda}(x)$ & 1 & $\varphi_{1 / \lambda}(y)$ \\
$c$ & 1 & $1-\varphi_{1 / \lambda}(y)$ & 1
\end{tabular}

The fuzzy relation $R$ is weakly complete and $T_{\mathrm{M}}$-transitive. For the corresponding strict preference relation generated by means of $T_{\lambda}^{\mathbf{F}}$ it holds that $P(a, b)=x, P(b, c)=y$ and $P(a, c)=0$. However, $P$ is not $g$-transitive, since

$$
P(a, c)=g^{\lambda}(x, y)<g(x, y)=g(P(a, b), P(b, c)) .
$$

(ii) If $\left(\varphi_{1 / \lambda}(x), \varphi_{1 / \lambda}(y)\right) \in \Delta$ and $y \leq \max \left(\varphi_{1 / \lambda}(x), 1-\varphi_{1 / \lambda}(x)\right)$, consider the reflexive fuzzy relation $R$ on $A=\{a, b, c\}$ given by

\begin{tabular}{c|ccc}
$R$ & $a$ & $b$ & $c$ \\
\hline$a$ & 1 & $\varphi_{1 / \lambda}(x)$ & $\varphi_{1 / \lambda}(x)$ \\
$b$ & $1-\varphi_{1 / \lambda}(x)$ & 1 & 1 \\
$c$ & $1-\varphi_{1 / \lambda}(x)$ & $1-y$ & 1
\end{tabular}

The fuzzy relation $R$ is weakly complete and $T_{\mathbf{M}}$-transitive. For the corresponding strict preference relation generated by means of $T_{\lambda}^{\mathbf{F}}$ it holds that $P(a, b)=x, P(b, c)=y$ and $P(a, c)=x$. However, $P$ is not $g$-transitive, since

$$
P(a, c)=g^{\lambda}(x, y)<g(x, y)=g(P(a, b), P(b, c)) .
$$

(iii) Finally, if $\left(\varphi_{1 / \lambda}(x), \varphi_{1 / \lambda}(y)\right) \in \Delta$ and $y>\max \left(\varphi_{1 / \lambda}(x), 1-\varphi_{1 / \lambda}(x)\right)$, consider the reflexive fuzzy relation $R$ on $A=\{a, b, c\}$ given by

\begin{tabular}{c|ccc}
$R$ & $a$ & $b$ & $c$ \\
\hline$a$ & 1 & $\varphi_{1 / \lambda}(x)$ & $y$ \\
$b$ & $1-\varphi_{1 / \lambda}(x)$ & 1 & 1 \\
$c$ & $1-y$ & $1-y$ & 1
\end{tabular}


The fuzzy relation $R$ is weakly complete and $T_{\mathbf{M}^{\mathbf{M}}}$-transitive. For the corresponding strict preference relation generated by means of $T_{\lambda}^{\mathbf{F}}$ it holds that $P(a, b)=x, P(b, c)=y$ and $P(a, c)=\varphi_{1 / \lambda}^{-1}(y)$. However, $P$ is not $g$-transitive, since

$$
P(a, c)=g^{\lambda}(x, y)<g(x, y)=g(P(a, b), P(b, c)) .
$$

Note that the conjunctor $g^{\lambda}$ does not have neutral element 1 and is therefore not a t-norm. Moreover, it does not hold that $g^{\lambda} \leq T_{\mathbf{M}}$. In the particular case $\lambda=1$, i.e. when using as generator $i=T_{\mathbf{P}}$, the above theorem expresses that

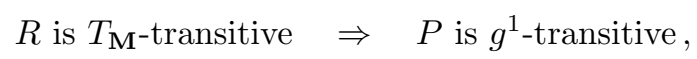

where

$$
g^{1}(x, y)= \begin{cases}0 & , \text { if } \sqrt{x}+\sqrt{y} \leq 1 \\ \min (x, y) & , \text { if } \sqrt{x}+\sqrt{y}>1 \\ \text { and } \max (x, & y) \leq \max (\sqrt{\min (x, y)}, 1-\sqrt{\min (x, y)}), \\ \max \left(x^{2}, y^{2}\right) & , \text { otherwise. }\end{cases}
$$

Theorem 5.4 For any weakly complete reflexive fuzzy relation $R$ with corresponding strict preference relation $P$ generated by means of $T_{\mathbf{M}}$, it holds that

$$
R \text { is } T_{\mathbf{M}} \text {-transitive } \Rightarrow P \text { is } g^{0} \text {-transitive, }
$$

where

$$
g^{0}(x, y)= \begin{cases}\min (x, y) & , \text { if } \min (x, y) \geq 2 \max (x, y)-1 \\ 2 \max (x, y)-1 & \text { or } \min (x, y)=0 \\ \text { otherwise }\end{cases}
$$

Moreover, this is the strongest result possible.

Proof. Let us verify that $g^{0}$ is a conjunctor. It is clear that $g^{0}$ takes values in $[0,1]$ and coincides on $\{0,1\}^{2}$ with the Boolean conjunction. Since $g^{0}(x, y)=0$ if $\min (x, y)=0$ and $g^{0}(x, y)=\max (\min (x, y), 2 \max (x, y)-1)$ otherwise, the increasingness of $g^{0}$ is immediate.

Next we prove the implication. Since Dasgupta and Deb [4] have already proven that $P$ is $T_{\mathbf{M}}$-transitive, and $g^{0} \geq T_{\mathbf{M}}$, it suffices to show that

$$
P(a, c) \geq 2 \max (P(a, b), P(b, c))-1
$$

whenever $\min (P(a, b), P(b, c))>0$. Assume, without loss of generality, that $P(a, b) \leq P(b, c)$. Since $P(a, c)=\max (R(a, c)-R(c, a), 0) \geq R(a, c)-R(c, a)$, it suffices to prove that

$$
R(a, c)-R(c, a) \geq 2 P(b, c)-1
$$


Since both $P(a, b)$ and $P(b, c)$ are strictly positive, it holds that $R(a, b)>R(b, a)$ and $R(b, c)>R(c, b)$. We now show that $R(c, b) \geq R(c, a)$. Due to the $T_{\mathbf{M}^{-}}$ transitivity of $R$, it holds that

$$
R(c, b) \geq \min (R(c, a), R(a, b)) .
$$

Suppose that $R(c, b)<R(c, a)$, whence $R(c, b) \geq R(a, b)$. It then follows that $R(c, b)>R(b, a) \geq \min (R(b, c), R(c, a))$. Since $R(b, a) \geq R(b, c)$ contradicts $R(b, c)>R(c, b)$, it holds that $R(b, a) \geq R(c, a)$, and hence also $R(c, b) \geq$ $R(c, a)$, again a contradiction. Therefore, $R(c, b) \geq R(c, a)$ effectively holds.

Since $R$ is weakly complete, it holds that $R(c, b) \geq R(c, a) \geq 1-R(a, c)$. Using $P(b, c)=R(b, c)-R(c, b) \leq 1-R(c, b)$, we find

$$
1-P(b, c) \geq R(c, b) \geq R(c, a) \geq 1-R(a, c),
$$

and conclude

$$
R(a, c)-R(c, a) \geq P(b, c)-(1-P(b, c))=2 P(b, c)-1 .
$$

It remains to be shown that no stronger result can be obtained. Consider a conjunctor $g$ such that $g(x, y)>g^{0}(x, y)$ for some $\left.\left.(x, y) \in\right] 0,1\right]^{2}$. Assume, without loss of generality, that $x \leq y$ and consider the reflexive fuzzy relation $R$ on $A=\{a, b, c\}$ given by

\begin{tabular}{c|ccc}
$R$ & $a$ & $b$ & $c$ \\
\hline$a$ & 1 & $(1-y)+g^{0}(x, y)$ & $(1-y)+g^{0}(x, y)$ \\
$b$ & $(1-x-y)+g^{0}(x, y)$ & 1 & 1 \\
$c$ & $1-y$ & $1-y$ & 1
\end{tabular}

The fuzzy relation $R$ is weakly complete and $T_{\mathbf{M}}$-transitive, but the corresponding strict preference relation generated by means of $T_{\mathbf{M}}$ is not $g$-transitive. Indeed, it holds that

$$
P(a, c)=g^{0}(x, y)<g(x, y)=g(P(a, b), P(b, c)) .
$$

Note that $g^{0}>T_{\mathbf{M}}$ and is therefore again not a t-norm. One easily verifies that Theorems 5.2-5.4 can be summarized as follows.

Corollary 5.5 For any weakly complete reflexive fuzzy relation $R$ with corresponding strict preference relation $P$ generated by means of $i=T_{\lambda}^{\mathbf{F}}, \lambda \in[0, \infty]$, it holds that

$$
R \text { is } T_{\mathbf{M}} \text {-transitive } \Rightarrow P \text { is } g^{\lambda} \text {-transitive, }
$$


where

$$
g^{\lambda}(x, y)= \begin{cases}0 & , \text { if } \varphi_{1 / \lambda}(x)+\varphi_{1 / \lambda}(y) \leq 1 \\ \min (x, y) & , \text { if } \varphi_{1 / \lambda}(x)+\varphi_{1 / \lambda}(y)>1 \\ \text { and } \max (x, y) \leq \max \left(\varphi_{1 / \lambda}(\min (x, y)), 1-\varphi_{1 / \lambda}(\min (x, y))\right), \\ \varphi_{1 / \lambda}^{-1}(\max (x, y)) & , \text { otherwise. }\end{cases}
$$

Moreover, these are the strongest results possible.

Note that $\lim _{\lambda \rightarrow 0} g^{\lambda}=g^{0}, \lim _{\lambda \rightarrow 1^{-}} g^{\lambda}=\lim _{\lambda \rightarrow 1^{+}} g^{\lambda}=g^{1}$ and $\lim _{\lambda \rightarrow \infty} g^{\lambda}=g^{\infty}$. Remarkably, the family $\left(g^{\lambda}\right)_{\lambda \in[0, \infty]}$ is neither increasing, nor decreasing w.r.t. the parameter $\lambda$. This is in contrast to the general case, where the t-norm family $\left(T_{\mathrm{nM}}^{\varphi_{1 / \lambda}}\right)_{\lambda \in[0, \infty]}$ describing the transitivity of $P$ is decreasing w.r.t. the parameter $\lambda$, from $T_{\mathbf{M}}$ to $T_{\mathbf{n M}}$.

\section{The strongly complete case revisited}

In this section we focus on the strongly complete case. From the characterization of $T$-transitive strongly complete fuzzy relations by De Baets et al. [10] (see also the next section), it follows in particular that the indifference and strict preference relations corresponding to a $T_{\mathrm{M}}$-transitive strongly complete fuzzy relation are also $T_{\mathrm{M}}$-transitive. However, this characterization does not reveal the strongest possible implications. Indeed, for the strict preference relation a stronger result can be obtained.

Theorem 6.1 For any strongly complete reflexive fuzzy relation $R$ with corresponding indifference and strict preference relations $I$ and $P$, it holds that

$$
R \text { is } T_{\mathbf{M}} \text {-transitive } \Rightarrow\left\{\begin{array}{l}
P \text { is } g_{\mathbf{M}} \text {-transitive, } \\
I \text { is } T_{\mathbf{M}} \text {-transitive, }
\end{array}\right.
$$

where

$$
g_{\mathbf{M}}(x, y)= \begin{cases}\max (x, y) & , \text { if } \min (x, y)>0, \\ 0 & , \text { otherwise. }\end{cases}
$$

Moreover, this is the strongest result possible.

Proof. As mentioned above, it was shown in [10] that $I$ is $T_{\mathbf{M}}$-transitive. Moreover, we have shown in [16] that in general the transitivity of a reflexive fuzzy relation is bounded by $T_{\mathbf{M}}$-transitivity. The same reasoning applies when considering only strongly complete fuzzy relations.

Next, we consider the strict preference relation. It suffices to consider $P(a, b)>0$ and $P(b, c)>0$ (which implies $R(b, a)<1$ and $R(c, b)<1$ ) and prove that

$$
\max (P(a, b), P(b, c)) \leq P(a, c) .
$$


Without loss of generality, we can assume that $P(a, b) \geq P(b, c)$. Since $R$ is strongly complete, it holds that $R(a, b)=1$ and $R(b, c)=1$, and $R(c, b) \geq$ $R(b, a)$. The $T_{\mathbf{M}^{-}}$-transitivity of $R$ implies that $R(a, c)=1$, as well as

$$
\min (R(b, c), R(c, a))=R(c, a) \leq R(b, a) .
$$

Hence, it holds that $P(a, c)=1-R(c, a) \geq 1-R(b, a)=P(a, b)$, from which it follows that $P(a, b)=\max (P(a, b), P(b, c)) \leq P(a, c)$.

It remains to be shown that no stronger result can be obtained. Consider a conjunctor $g$ satisfying $g(x, y)>g_{\mathbf{M}}(x, y)$ for some $\left.\left.(x, y) \in\right] 0,1\right]^{2}$. Assume, without loss of generality, that $x \leq y$ and consider the following reflexive fuzzy relation $R$ on $A=\{a, b, c\}$ given by

\begin{tabular}{c|ccc}
$R$ & $a$ & $b$ & $c$ \\
\hline$a$ & 1 & 1 & 1 \\
$b$ & $1-x$ & 1 & 1 \\
$c$ & $1-y$ & $1-y$ & 1
\end{tabular}

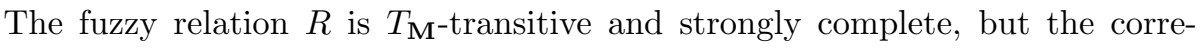
sponding strict preference relation $P$ is not $g$-transitive, since

$$
P(a, c)=y=g_{\mathbf{M}}(x, y)<g(x, y)=g(P(a, b), P(b, c)) .
$$

As was the case when considering a weakly complete instead of a general reflexive fuzzy relation $R$, also the transitivity that can be guaranteed for $P$ is stronger when considering a strongly complete instead of a weakly complete reflexive fuzzy relation $R$. More in particular, it holds that $g_{\mathbf{M}}>g^{\lambda}$, for any $\lambda \in[0, \infty]$; this is not surprising as the choice of the generator is irrelevant in the strongly complete case.

\section{On the converse implication}

The only generalization of Theorems 1 and 2 to the fuzzy setting is due to De Baets et al. [10]: for a strongly complete fuzzy relation $R$ and a t-norm $T \geq T_{\mathbf{L}}$ it holds that

$R \circ_{T} R \subseteq R \Leftrightarrow\left(P \circ_{T_{\mathbf{M}}} P \subseteq P \wedge I \circ_{T} I \subseteq I \wedge P \circ_{T_{\mathbf{L}}} I \subseteq P \wedge I \circ_{T_{\mathbf{L}}} P \subseteq P\right)$.

This result is intermediate between Theorems 1 and 2: although we are dealing with a strongly complete fuzzy relation, the mixed compositional inequalities enter as conditions in the converse implication. Note that this characterization does not require the strongest type of transitivity of $P$, namely $g_{\mathbf{M}}$-transitivity.

In this section, we will try to generalize the above result for $T=T_{\mathbf{M}}$ and a weakly complete fuzzy relation. Moreover, as the strongest type of transitiv-

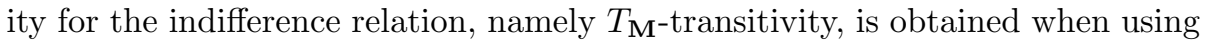


$i=T_{\mathrm{M}}$ (Corollary 4.5), we will focus our attention on this generator. Definitely, a generalization will require stronger conditions on the right-hand side. As the strongest transitivity condition for $P$, namely $g^{0}$-transitivity, has been determined in Section 5, we investigate the mixed compositional inequalities.

Proposition 7.1 For any weakly complete reflexive fuzzy relation $R$ with corresponding indifference and strict preference relations $I$ and $P$ generated by means of $i=T_{\mathbf{M}}$, it holds that

$$
R \circ_{T_{\mathbf{M}}} R \subseteq R \Rightarrow\left(P \circ_{T_{\mathbf{L}}} I \subseteq P \wedge I \circ_{T_{\mathbf{L}}} P \subseteq P\right) .
$$

Moreover, this is the strongest result possible.

Proof. We prove the first inclusion, the second one being analogous. Consider $(a, b, c) \in A^{3}$, then we have to show that

$$
\max (P(a, b)+\min (R(b, c), R(c, b))-1,0) \leq P(a, c),
$$

or equivalently,

$$
\min (P(a, b)+R(b, c)-1, P(a, b)+R(c, b)-1) \leq P(a, c) .
$$

It suffices to prove that $P(a, b)+R(b, c)-1 \leq P(a, c)$. In case $P(a, b)=0$, this inequality trivially holds. In case $P(a, b)>0$, it holds that $P(a, b)=$ $R(a, b)-R(b, a)$. We distinguish two cases:

(i) If $R(b, a) \geq R(b, c)$, then

$$
P(a, b)+R(b, c)-1=R(a, b)-R(b, a)+R(b, c)-1 \leq 0 \leq P(a, c) .
$$

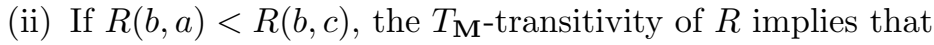

$$
\begin{aligned}
& R(b, a) \geq \min (R(b, c), R(c, a))=R(c, a) \\
& R(a, c) \geq \min (R(a, b), R(b, c)) \geq R(a, b)+R(b, c)-1 .
\end{aligned}
$$

It then follows that

$$
\begin{aligned}
P(a, c) & =\max (R(a, c)-R(c, a), 0) \\
& \geq R(a, c)-R(c, a) \\
& \geq R(a, b)+R(b, c)-1-R(b, a) \\
& =P(a, b)+R(b, c)-1 .
\end{aligned}
$$

No stronger result can be obtained. Consider a conjunctor $h$ such that $h(x, y)>T_{\mathbf{L}}(x, y)$ for some $\left.\left.(x, y) \in\right] 0,1\right]^{2}$. Consider the reflexive fuzzy relation $R$ on $A=\{a, b, c\}$ given by

\begin{tabular}{c|ccc}
$R$ & $a$ & $b$ & $c$ \\
\hline$a$ & 1 & 1 & $y$ \\
$b$ & $1-x$ & 1 & $y$ \\
$c$ & $1-x \chi_{\Delta}(x, y)$ & 1 & 1
\end{tabular}


The fuzzy relation $R$ is weakly complete and $T_{\mathrm{M}}$-transitive, but the strict preference and indifference relations generated by means of $i=T_{\mathbf{M}}$ do not satisfy $P \circ_{h} I \subseteq P$, since

$$
P(a, c)=T_{\mathbf{L}}(x, y)<h(x, y)=h(P(a, b), I(b, c)) .
$$

Similarly, consider a conjunctor $g$ such that $g(x, y)>T_{\mathbf{L}}(x, y)$ for some $(x, y) \in] 0,1]^{2}$. Consider the reflexive fuzzy relation $R$ on $A=\{a, b, c\}$ given by

\begin{tabular}{c|ccc}
$R$ & $a$ & $b$ & $c$ \\
\hline$a$ & 1 & $x$ & $x$ \\
$b$ & 1 & 1 & 1 \\
$c$ & $1-y \chi_{\Delta}(x, y)$ & $1-y$ & 1
\end{tabular}

The fuzzy relation $R$ is weakly complete and $T_{\mathrm{M}}$-transitive, but the strict preference and indifference relations generated by means of $i=T_{\mathbf{M}}$ do not satisfy $I \circ_{h} P \subseteq P$, since

$$
P(a, c)=T_{\mathbf{L}}(x, y)<h(x, y)=h(I(a, b), P(b, c)) .
$$

Example 7.1 Consider the weakly complete reflexive fuzzy relation $R$ on $A=$ $\{a, b, c\}$ given by

\begin{tabular}{c|ccc}
$R$ & $a$ & $b$ & $c$ \\
\hline$a$ & 1 & 1 & 0.8 \\
$b$ & 0.7 & 1 & 1 \\
$c$ & 0.5 & 0.5 & 1
\end{tabular}

The strict preference and indifference relations generated by means of $i=T_{\mathbf{M}}$ are given by

\begin{tabular}{c|ccc}
$P$ & $a$ & $b$ & $c$ \\
\hline$a$ & 0 & 0.3 & 0.3 \\
$b$ & 0 & 0 & 0.5 \\
$c$ & 0 & 0 & 0
\end{tabular}

\begin{tabular}{c|ccc}
$I$ & $a$ & $b$ & $c$ \\
\hline$a$ & 1 & 0.7 & 0.5 \\
$b$ & 0.7 & 1 & 0.5 \\
$c$ & 0.5 & 0.5 & 1
\end{tabular}

One easily verifies that $P$ is $g^{0}$-transitive, $I$ is $T_{\mathbf{M}}$-transitive and $P \circ_{T_{\mathbf{L}}} I \subseteq P$ and $I \circ_{T_{\mathrm{L}}} P \subseteq P$. However, the fuzzy relation $R$ does not even satisfy the weakest type of transitivity ( $c_{S}$-transitivity), since

$$
R(a, c)=0.8<c_{S}(1,1)=c_{S}(R(a, b), R(b, c)) .
$$

We therefore conclude that

$$
R \circ_{c_{S}} R \subseteq R \quad \nLeftarrow \quad\left\{\begin{array}{l}
P \circ_{g^{0}} P \subseteq P \\
I \circ_{T_{\mathrm{M}}} I \subseteq I \\
P \circ_{T_{\mathrm{L}}} I \subseteq P \\
I \circ_{T_{\mathrm{L}}} P \subseteq P .
\end{array}\right.
$$

This is a rather disappointing result as it implies that characterizations in terms of fuzzy relational compositions are simply not possible, even not in the weakly complete case. 


\section{Conclusion}

In this work, we have carried out an in-depth study of the transitivity of the indifference and strict preference relations generated from a strongly or weakly complete fuzzy pre-order. In the weakly complete case, we have identified the strongest type of transitivity of the indifference relation in function of the generator, while for the strict preference relation this has (only) been realized for a Frank t-norm as generator, leading to an interesting family of conjunctors. Moreover, in the strongly complete case, we have identified the strongest type of transitivity of the strict preference relation. Finally, we have shown that the quest for compositional characterizations of fuzzy pre-orders, other than in the known strongly complete case, can never be successful.

\section{Acknowledgements}

The research reported on in this paper has been partially supported by Projects FEDER-MEC-MTM2004-01269 and FEDER-MEC-MTM2007-61193.

\section{References}

[1] K. J. Arrow, Social Choice and Individual Values, Wiley, 1951.

[2] U. Bodenhofer, B. De Baets, J. Fodor, A compendium of fuzzy weak orders: representations and characterizations, Fuzzy Sets and Systems 158 (2007), 811-829.

[3] U. Bodenhofer, F. Klawonn, A formal study of linearity axioms for fuzzy orderings, Fuzzy Sets and Systems 145 (2004), 323-354.

[4] M. Dasgupta, R. Deb, Factoring fuzzy transitivity, Fuzzy Sets and Systems 118 (2001), 489-502.

[5] B. De Baets, S. Janssens and H. De Meyer, On the transitivity of a parametric family of cardinality-based similarity measures, Internat. J. Approximate Reasoning, in press.

[6] B. De Baets, J. Fodor, Additive fuzzy preference structures: the next generation, in: B. De Baets, J. Fodor (Eds.), Principles of Fuzzy Preference Modelling and Decision Making, Academia Press, 2003, pp. 15-25.

[7] B. De Baets, B. Van De Walle, Minimal definitions of classical and fuzzy preference structures, Proc. Annual Meeting of the North American Fuzzy Information Processing Society, Syracuse, New York, USA, 1997, pp. 299304.

[8] B. De Baets, H. De Meyer, H. Naessens, A top-down algorithm for generating the Hasse tree of a fuzzy preorder closure, IEEE Trans. Fuzzy Systems 12 (2004), 838-848. 
[9] B. De Baets, J. Fodor, E. E. Kerre, Gödel representable fuzzy weak orders, Internat. J. Uncertain. Fuzziness Knowledge-Based Systems 7 (1999), 135154.

[10] B. De Baets, B. Van De Walle, E. Kerre, Fuzzy preference structures without incomparability, Fuzzy Sets and Systems 76 (1995), 333-348.

[11] B. De Baets, B. Van de Walle, E. Kerre, A plea for the use of Łukasiewicz triplets in the definition of fuzzy preference structures. Part 2: The identity case, Fuzzy Sets and Systems 99 (1998), 303-310.

[12] H. De Meyer, H. Naessens, B. De Baets, Algorithms for computing the min-transitive closure and associated partition tree of a symmetric fuzzy relation, European J. Oper. Res. 155 (2004), 226-238.

[13] S. Díaz, B. De Baets, S. Montes, On the transitivity of fuzzy indifference relations, Lecture Notes in Artificial Intelligence (Subseries of Lecture Notes in Computer Science) 2715 (2003), 87-94.

[14] S. Díaz, B. De Baets, S. Montes, Additive decomposition of fuzzy preorders, Fuzzy Sets and Systems 158 (2007), 830-842.

[15] S. Díaz, S. Montes, B. De Baets, Transitive decomposition of fuzzy preference relations: the case of nilpotent minimum, Kybernetika 40 (2004), 71-88.

[16] S. Díaz, S. Montes, B. De Baets, Transitivity bounds in additive fuzzy preference structures, IEEE Trans. Fuzzy Systems 15 (2007), 275-286.

[17] J. Fodor, Contrapositive symmetry of fuzzy implications, Fuzzy Sets and Systems 69 (1995), 141-156.

[18] J. Fodor, M. Roubens, Fuzzy Preference Modelling and Multicriteria Decision Support, Kluwer Academic Publishers, 1994.

[19] C. Genest, J.J. Quesada-Molina, J.A. Rodríguez-Lallena, C. Sempi, A characterization of quasi-copulas, Journal of Multivariate Analysis 69 (1999), 193-205.

[20] U. Höhle, N. Blanchard, Partial ordering in L-underdeterminate sets, Information Sciences 35 (1985), 133-144.

[21] S. Janssens, B. De Baets, H. De Meyer, Bell-type inequalities for quasicopulas, Fuzzy Sets and Systems 148 (2004), 263-278.

[22] E.P. Klement, R. Mesiar, E. Pap, Triangular Norms, Kluwer Academic Publishers, 2000.

[23] K.C. Maes and B. De Baets, On the structure of left-continuous t-norms that have a continuous contour line, Fuzzy Sets and Systems 158 (2007), 843-860. 
[24] R.B. Nelsen, An Introduction to Copulas, 2nd ed., Springer, New York, 2006.

[25] M. Roubens, Ph. Vincke, Preference modelling, Lecture Notes in Economics and Mathematical Systems, Vol. 76, Springer-Verlag, 1985.

[26] B. Van de Walle, B. De Baets, E. Kerre, A plea for the use of Łukasiewicz triplets in the definition of fuzzy preference structures. Part 1: General argumentation, Fuzzy Sets and Systems 97 (1998), 349-359.

[27] B. Van de Walle, B. De Baets, E. Kerre, Characterizable fuzzy preference structures, Ann. Oper. Res. 80 (1998), 105-136.

[28] L. Zadeh, Similarity relations and fuzzy orderings, Information Sciences $\mathbf{3}$ (1971), 177-200. 